\title{
Tris-Benzamide Analogs for Inhibiting Bcl-2 Proteins in Prostate Cancer
}

\author{
Joongsoo Kim, Rakesh Kumar, and Jung-Mo Ahn* \\ Department of Chemistry and Biochemistry, University of Texas at Dallas, Richardson, TX, 75080, USA
}

\section{Introduction}

Apoptosis is an important cellular mechanism for tissue homeostasis and aberration in the process is well documented in cancers including castration-resistant prostate cancer (CRPC) [1]. Since it is regulated by heterodimerization of $\mathrm{Bcl}-2$ family proteins, disrupting such protein complexes is attractive for therapeutic intervention. Structural studies revealed that the helical BH3 domain of pro-apoptosis proteins including Bak binds to a hydrophobic pocket in anti-apoptotic proteins like Bcl-xL $[2,3]$. To mimic helical BH3 domain, we have reported the synthesis and biological activity of tris-benzamides which can place three functional groups corresponding to the side chains found at the $i, i+4$, and $i+7$ positions in an $\alpha$-helix [4,5]. To improve the activity of tris-benzamides, we have modified them by introducing additional side chain group at either end of molecules or changing one of side chain group. This library of tris-benzamide analogs as BH3 peptidomimetics was then examined for their binding affinity to anti-apoptotic Bcl-xL protein, inhibition of cell proliferation of various prostate cancer cell lines, mechanism of action, and in vivo efficacy. We have identified several leading compounds that showed strong binding affinity as well as high metabolic stability and cell permeation. These compounds were found to be effective in inhibiting cell growth of several prostate cancer cell lines and provided evidence of inducing apoptosis. These results show a potential of BH3 mimetics in treating prostate cancer.

\section{Results and Discussion}

We have designed and synthesized a number of tris-benzamides as peptidomimetics of BH3 domains of pro-apoptotic Bcl-2 proteins like Bak, Bad, Bim, Bik, Bid, Puma, and Noxa, and several compounds were found to be capable of inhibiting Bcl-xL from making a complex with Bak. To increase their potency, we have derivatized one of the leading compounds. We have introduced a variety of functional groups at each end of the molecule in order to improve its inhibitory potency. Among many functional groups surveyed, a carboxylate either at the $N$ - or $C$-terminus was found to be the most effective.

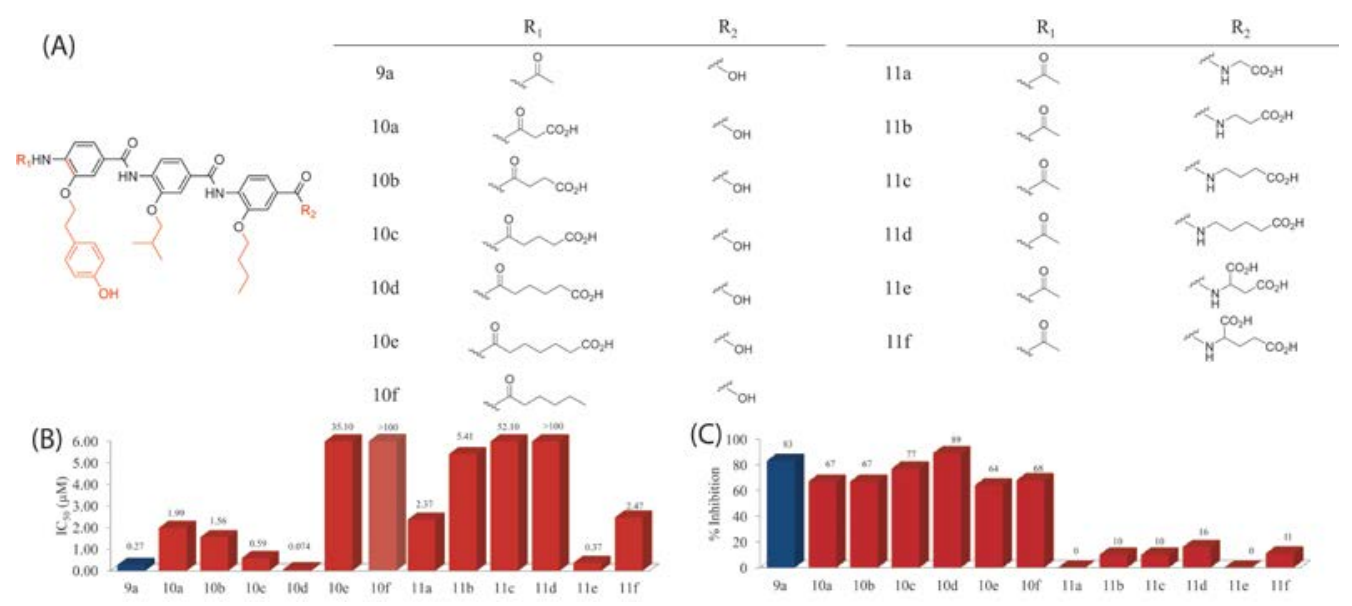

Fig. 1. (A) Structures of structural analogs of BH3 peptidomimetics and (B) their binding affinity to Bcl-xL and (C) cytotoxicity on a prostate cancer cell line DU145. 


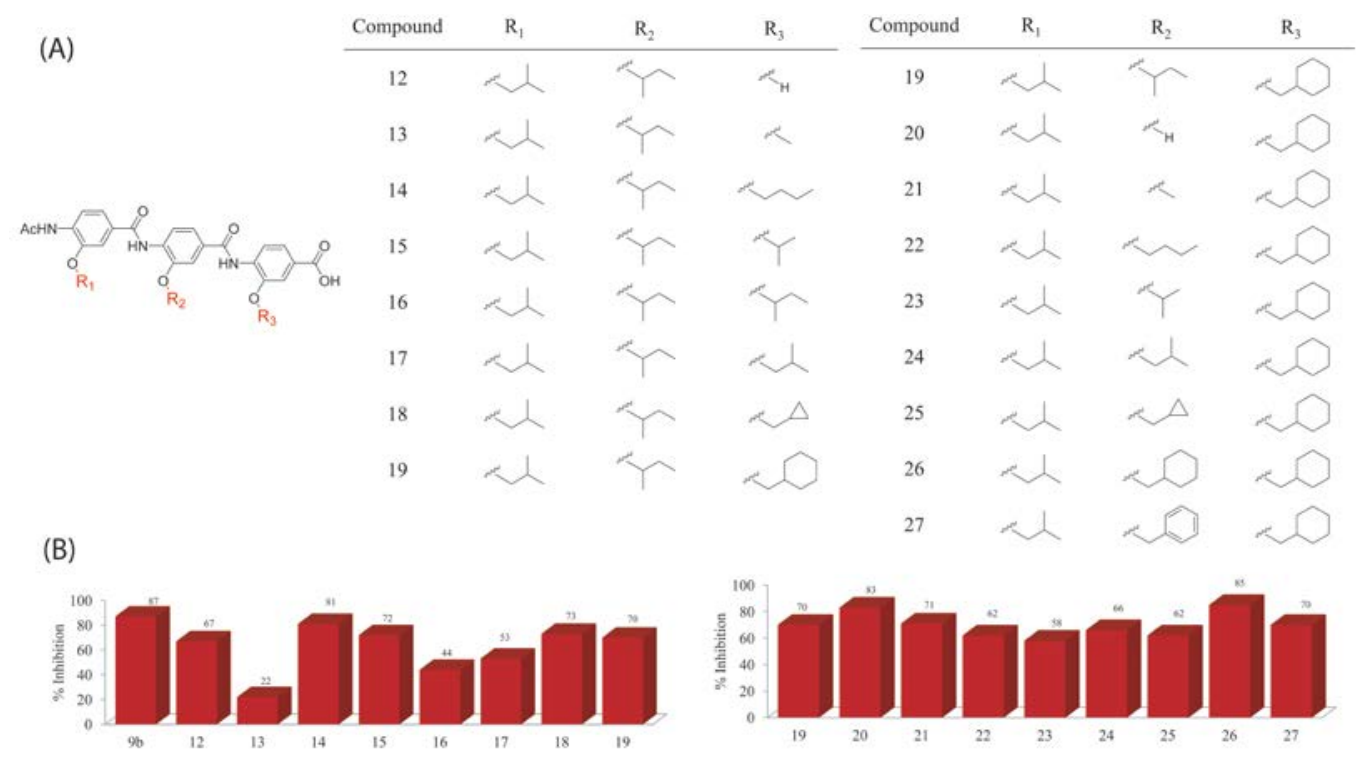

Fig. 2. (A) Selected structures of small chemical library of tris-benzamides and (B) their cytotoxicity on a prostate cancer cell line DU145.

In addition, we also constructed a small chemical library of tris-benzamides. Three substituents of the tris-benzamide scaffold were varied to create topologically diverse hydrophobic surfaces of an $\alpha$-helix. A number of tris-benzamides were synthesized and tested on protein binding assays on Bcl-xL and cytotoxicity on a prostate cancer cell line DU145. Several potent compounds were identified for high cell proliferation inhibition as shown in Figure 2.

\section{Acknowledgments}

This work was supported in part by the Cancer Prevention and Research Institute of Texas (RP100718) and the Welch Foundation (AT-1595).

\section{References}

1. Thompson, C.B. Science 267, 1456-1462 (1995), http://dx.doi.org/doi:10.1126/science.7878464

2. Liu, X., et al. Immunity 19, 341-352 (2003), http://dx.doi.org/doi:10.1016/S1074-7613(03)00234-6

3. Petros, A.M., et al. Protein Sci. 9, 2528-2534 (2000), http://dx.doi.org/10.1110/ps.9.12.2528

4. Ahn. J.-M., Han, S.-Y. Tetrahedron Lett. 48, 3543-3547 (2007), http://dx.doi.org/10.1016/j.tetlet.2007.03.108

5. Marimganti, S., et al. Org. Lett. 11, 4418-4421 (2009), http://dx.doi.org/10.1021/ol901785v 